\title{
Pyogenic granuloma of gingiva - A case report
}

\author{
Monica $^{1, *}$, Dakshaini' ${ }^{2}$, Rajhavee ${ }^{3}$, Salome $^{4}$, MSM Biir $^{5}$ \\ ${ }^{1}$ PG Student, ${ }^{2,3,4}$ Oral Pathologist, ${ }^{5} \mathrm{HOD},{ }^{1,5}$ Maharaja Ganga Singh Dental College \& Research Centre, Ganganagar, Rajasthan, \\ ${ }^{2}$ Angel Dental Clinic, ${ }^{4}$ Dental Clinician
}

Corresponding Author:

Email: monica.gs@rediffmail.com

\begin{abstract}
One of the most commonly seen swiftly increasing, highly reactive proliferation of endothelial cells affecting the gingival tissue and usually happens in response to a chronic irritation is known as Pyogenic Granuloma. The current study reports a case of pyogenic granuloma located on the lingual (inner) surface of the gingiva in the left mandibular region that was managed by surgical intervention.
\end{abstract}

Keywords: Pyogenic Granuloma, Pregnancy Tumor, Female Sex Hormones.

\section{Introduction}

Fibrous growths of the oral cavity are very common including reactive group and neoplastic conditions. ${ }^{1}$ Among these, pyogenic granuloma is localized granulation tissue found either in the oral cavity or on the skin but is labeled as non - neoplastic. The term "Pyogenic granuloma" (PG) or "granuloma pyogenicum" was familiarized by Hartzell in the year $1904^{2}$ Though a misnomer in itself as there is no production of pus, it does characterize a granulomatous inflammation. ${ }^{3,4}$ Looking into the etiology, it is seen that there exists no co - relation between any of the bacteria and appearance of this specific proliferation. ${ }^{3,4}$

It is actually a benign, though hyperactive lesion that is seen commonly inside the oral cavity. The most commonly affected site is the gingiva, then followed by the buccal mucosa, tongue and lips. ${ }^{5}$ It arises in response to various stimuli such as low grade local irritation, traumatic injury, hormonal factors, or to certain kinds of drugs (eg. Retinoid ,antiretroviral drug,oncologic agent subtypes). ${ }^{6}$ Pyogenic granuloma may occur in all age groups, though it is predominantly seen in young females in the second decade of life because it is believed that female sex hormones play an important role in its pathogenesis. ${ }^{5} \mathrm{~A}$ case of pyogenic granuloma which was successfully managed by surgical intervention is presented here with.

\section{Case Report}

A 42 years old female patient reported to the Department of Periodontics and Implantology, Maharaja Ganga Singh Dental College and Research Centre, Sri Ganganagar, with chief complaint of swelling in the inter proximal gingival tissue lingually in relation to left side of lower jaw. Patient noticed the lesion about 4 months back. As per the patient, this lesion was initially small in size and has gradually increased to the present dimensions. Further, patient had discomfort while chewing the food on the left side and found it difficult to maintain the oral hygiene.
Intraoral examination revealed a smooth pedunculated exophytic hemorrhagic lesion which bled on slightest provocation. It extended from mesial aspect of mandibular left canine (\#33) to distal aspect of the mandibular left first premolar (\#34) and was $2 \times 4 \mathrm{~cm}$ in size. [Fig. 1] The growth was firm on palpation, non tender with absence of any discharge. There was mild to moderate amount of calculus. Further there was no relevant past medical and dental history. Complete hemogram showed all blood counts to be within normal limits. Radiographic examination showed no alveolar bone involvement.

Oral prophylaxis was carried out. Patient was prescribed $0.2 \%$ chlorhexidine gluconate mouthwash twice daily. Under local anesthesia, using \#15 blade, the entire lesion was excised from the underlying tissue [Fig. 2] and the bleeding was controlled by pressure pack. [Fig. 3] The excised tissue was sent for histological examination which revealed parakeratinised stratified squamous epithelium with hyperplasia. Connective tissue showed blood vessels with engorged RBC's, budding capillaries and proliferation of endothelial cells. Chronic inflammatory cell infiltrate was mainly composed of lymphocytes, plasma cells, fibroblasts and collagen fibers.

Histological and clinical examination when correlated, were suggestive of pyogenic granuloma. The patient was recalled after a week and the excised area healed uneventfully with a return to normal physiological contour. The patient was recalled periodically and oral prophylaxis was done. Along with this, oral hygiene instructions were reinforced at each visit. 


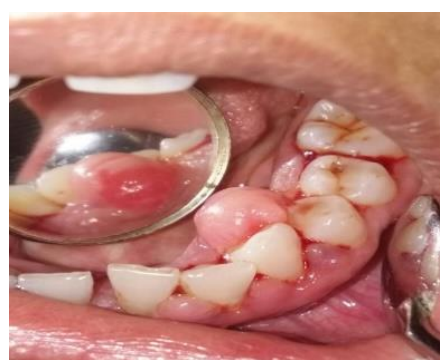

Fig. 1

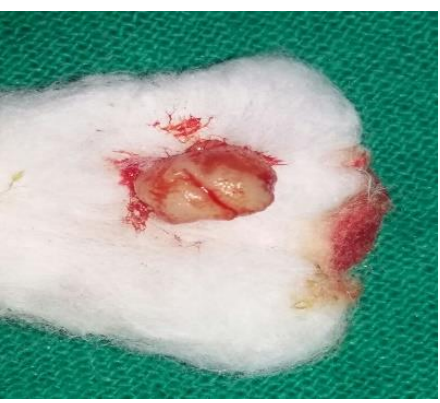

Fig. 2

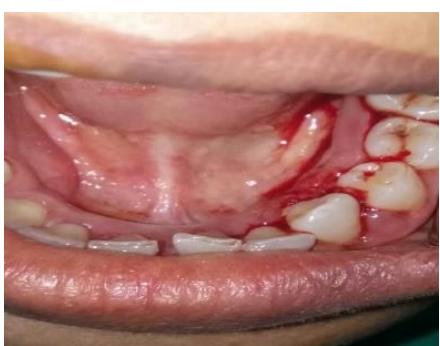

Fig. 3

\section{Discussion}

Gingival enlargement is defined as an increase in size of the gingiva depending on the etiologic factors, it can be of many types like inflammatory, drug induced effects, neoplasm, hormonal imbalance, nonspecific conditioned enlargement and in many systemically involving diseases like Leukemias, Wegener's granulomatosis, etc. ${ }^{6}$

Poncet and Dor in the year 1897 had first described pyogenic granuloma as granuloma pyogenicum. Over the years, various authors have suggested many other names such as granuloma gravidarum/pregnancy tumour, hemangiomatosis granuloma, Crocker and Hartzell's disease, benign vascular tumour, vascular epulis, epulis teleangiectaticum granulomatosa, lobular capillary hemangioma. ${ }^{7,8}$

Morphologically, it appears as smooth pedunculated exophytic hemorrhagic lesion of deep red in color, highly vascular and soft in consistency and similar findings are reported by Mohapatra et al. ${ }^{16}$ Patel $\mathrm{K}$ et $\mathrm{al}^{10}$ described the morphological features of pyogenic granuloma as usually an elevated, pedunculated or sessile vascular mass with a smooth, lobulated or even warty surface. It ranges from a few millimeters to more in diameter and commonly is ulcerated and shows tendency for hemorrhage either spontaneously or upon slight trauma and it commonly occurs in facial aspect than in lingual or palatal aspect and can involve both sides including interdental papilla.

Histologically, the lesion revealed parakeratinised stratified squamous epithelium with hyperplasia. Connective tissue showed blood vessels with engorged RBC's, budding capillaries and proliferation of endothelial cells. Chronic inflammatory cell infiltrate was mainly composed of lymphocytes, plasma cells, fibroblasts and collagen fibers. Similar results were reported by Mohapatra et al, ${ }^{16}$ Shafers et al. ${ }^{9}$

Pregnancy tumor is histologically identical to pyogenic granuloma of the gingiva, it frequently occurs during pregnancy and is often called as pregnancy tumor or pregnancy granuloma Gravidarum. This is a well defined lesion which occur in third month of pregnancy or sometime later, gradually increases in size and after delivery, may or may not regress. ${ }^{9}$ If surgically removed during pregnancy, it frequently recurs. It is believed that pregnancy tumor is simply a pyogenic granuloma which occurs as a result of local minor trauma or irritation and in which the tissue reaction is probably intensified by the endocrine alteration occurring during pregnancy. ${ }^{9}$

Although pyogenic granuloma can be diagnosed clinically with considerable accuracy, radiographic and histopathological investigations aid in confirming the diagnosis and treatment is advised to rule out any bony destructions and soft tissue changes. ${ }^{11}$

Differential diagnosis of PG includes parulis, peripheral giant cell granuloma, peripheral ossifying fibroma, hemangioma, peripheral fibroma, leiomyoma, hemangioendothelioma, hemangiopericytoma, bacillary angiomatosis, Kaposi's sarcoma, metastatic tumor, post extraction granuloma and pregnancy tumor. ${ }^{12}$

Surgical excision is the treatment of choice. After surgical excision of gingival lesions, curettage of underlying tissue is recommended. ${ }^{13}$ Laser therapy using continuous and pulsed $\mathrm{CO}_{2}$ and Nd-YAG may be performed because it is less invasive and suture less procedure that produces only minimal post-operative pain. ${ }^{14}$ Incomplete excision, failure to remove etiologic factors or repeated trauma contributes to recurrence of these lesions. ${ }^{9,15}$

\section{Conclusion}

Pyogenic granuloma is a fast growing reactive proliferation of endothelial cells commonly on the gingiva and usually in response to chronic irritation. It is a polypoid form of capillary hemangioma on the skin $\&$ mucosal surfaces. The present case report represents recognition, identification, clinical assessment as well as dental management of patient with pyogenic granuloma. 


\section{References}

1. Kotle AP, Kolte RA, Shriro TS. Focal fibrous overgrowth; A case series and review of literature. Contemp Clin Dent 2010;1(4);271-274.

2. Jafarzadeh H, Sanatkhani M, Mohtasham N. Oral pyogenic granuloma: a review. J Oral Sci 2006;48(4):167-75

3. Sharon W. Weies, John R Goldblum. Enzinger's \& Weiss's soft tissue tumors 864-865.

4. Neville, Damm, Allen bouquet. Oral\& maxillofacial pathology;447-449.

5. Bhaskar SN, Jacoway JR. Pyogenic granulom: clinical features, incidence, histology, and result of treatment: report of 242 cases. J Oral Surg 1966;24(5):391-8.

6. Sumanth Shivaswamy, Nazia Siddiqui, A. Sanjay Jain, Ajit Koshy, Sonal Tambwekar, Akhil Shankar. A rare case of generalized pyogenic granuloma: A case report. Quintessence Int 2011;42:493-499.

7. Graham RM: Pyogenic granuloma: an unusual presentation, Dental Update 1996;26(1):240-1.

8. Bjork K, Hoede N, Korting GW, Burgdorf WHC, Young SK: Diseases of the oral mucosa and the lips. WB Saunders, Philadelphia, 1996:229-30

9. Shafer, Hine \& Levy. Textbook of oral pathology 2006

10. Patil K, Mahima VG, Lahari K. Extragingival pyogenic granuloma. Indian J Dent Res 2006;17:199-202.

11. Shenoy SS, Dinkar AD. Pyogenic granuloma associated with bone loss in an eight year oldchild: A case report. J Indian SocPedodPrev Dent 2006 December: 201-3.

12. Wood NK, Goaz PW. Differential diagnosis of oral and maxillofacial lesions. 5 th ed. Missouri: Mosby; 1998. p. 129

13. Patil K, Mahima VG, Lahari K. Extragingival pyogenic granuloma. Indian J Dent Res 2006;17:199-202.

14. Khandpur S,Sharma VK. Indian J Dermatol Venereol Leprol.2008;74(3):275-277

15. Sharon W. Weies, John R Goldblum. Enzinger's \& Weiss's soft tissue tumors 864-865.

16. Mohapatra S, Binjoo N, Arora KS, Modgil R. Oral Pyogenic Granuloma: Series of 3 Case Reports. Journal of Dentofacial Sciences, 2014; 3(3): 23-26. 